\title{
ON NONOVERLAPPING DOMAIN DECOMPOSITION METHODS FOR THE INCOMPRESSIBLE NAVIER-STOKES EQUATIONS*
}

\author{
Xuejun Xu ${ }^{1}$, C.O. ChOw $^{2}$ And S.H. LuI ${ }^{3}$
}

\begin{abstract}
In this paper, a Dirichlet-Neumann substructuring domain decomposition method is presented for a finite element approximation to the nonlinear Navier-Stokes equations. It is shown that the Dirichlet-Neumann domain decomposition sequence converges geometrically to the true solution provided the Reynolds number is sufficiently small. In this method, subdomain problems are linear. Other version where the subdomain problems are linear Stokes problems is also presented.
\end{abstract}

Mathematics Subject Classification. 65F10, 65N30, 65N55.

Received: April 1, 2004.

\section{INTRODUCTION}

With the development of parallel computers, domain decomposition methods (DDMs) have become increasingly important tools for solving PDEs. In general, there exist two kinds of domain decomposition methods. One is the overlapping Schwarz method which is often referred to as the Schwarz alternating method $[3,12,13,15,18]$ and the other is the class of nonoverlapping domain decomposition methods. The distinction is whether the domain is decomposed into overlapping or nonoverlapping subdomains. In this paper, we shall focus our attention on nonoverlapping domain decomposition methods. These DDMs have been studied extensively for linear PDEs, see $[11,17,20]$ and the references therein. The literature for nonlinear problems is rather sparse. The efficiency of DDMs for the Navier-Stokes equations has already been demonstrated by many authors. See $[1,2,5-7,10]$ for just a small sample. Recently, a so-called optimization-based DDM has been proposed in [9]. It is shown that this DDM is convergent, but no convergence rate is given. See the references in the books and review articles mentioned above and the proceedings of the DDM conferences for many more references.

This paper seems to be the first attempt to give a convergence rate for a nonoverlapping DDM for nonlinear equations. We shall present a Dirichlet-Neumann (D-N) iterative substructuring algorithm for a finite element approximation to the Navier-Stokes (N-S) equations. For sufficiently small Reynolds number, we prove that the

\footnotetext{
Keywords and phrases. Nonoverlapping domain decomposition, incompressible Navier-Stokes equations, finite elements, nonlinear problems.

* This work is subsidized by the special funds for major state basic research projects under 2005 CB321701 and a grant from the National Science Foundation (NSF) of China (No. 10471144). This work was in part supported by a grant from NSERC of Canada.

${ }^{1}$ LSEC, Institute of Computational Mathematics, Academy of Mathematics and System Sciences, Chinese Academy of Sciences, PO Box 2719, Beijing, 100080, China. xxj@lsec.cc.ac.cn

2 Institute of Mathematics, Academia Sinica, Taipei 11529, Taiwan. cchow@alum.mit.edu

3 Department of Mathematics, University of Manitoba, Winnipeg, Manitoba, R3T 2N2, Canada. luish@cc.umanitoba.ca
} 
D-N substructuring method is optimal, i.e., the convergence rate of this method is independent of the mesh size. Because the Reynolds number is small, the N-S equations are just a small perturbation of the Stokes equations. Our method of proof relies heavily on this fact and we follow the framework developed in [16].

For our method, the subdomain problems are linear equations. We shall also present a linear D-N algorithm where the subdomain problems are linear Stokes equations. We also discuss a Neumann-Neumann (N-N) iterative substructuring method for the N-S equations. In [14], some overlapping Schwarz algorithms were developed for the N-S equations. This paper can be regarded as a continuation of [14] for the nonoverlapping case.

This paper is organized as follows. In Section 2, we shall introduce the model problem and its corresponding finite element approximation. A D-N iterative algorithm for this discrete system will be given in Section 3 . Other linear D-N iterative algorithms will be considered in Section 4 followed by a short discussion on a N-N algorithm in Section 5. In Section 6, we illustrate the behavior of the linear D-N algorithms for a flow in a square.

\section{Model PROBLEM}

We consider the following N-dimensional, steady, viscous incompressible Navier-Stokes equations:

$$
\left\{\begin{aligned}
-\frac{1}{R_{e}} \Delta u+(u \cdot \nabla) u+\nabla p & =f, & & \text { on } \Omega, \\
\operatorname{div} u & =0, & & \text { on } \Omega, \\
u & =0, & & \text { on } \partial \Omega,
\end{aligned}\right.
$$

where $\Omega$ is a convex polyhedral domain in $R^{N}(N \leq 3), u \in\left(H_{0}^{1}(\Omega)\right)^{N}, f$ is a forcing term in $\left(L^{2}(\Omega)\right)^{N}, p$ is the pressure and $R_{e}$ is the Reynolds number.

Let $V=\left(H_{0}^{1}(\Omega)\right)^{N}$, and

$$
M=L_{0}^{2}(\Omega)=\left\{q \in L^{2}(\Omega) \mid \int_{\Omega} q \mathrm{~d} x=0\right\} .
$$

The variational formula of (2.1) is to find $u \in V$ and $p \in M$ such that

$$
\left\{\begin{aligned}
\frac{1}{R_{e}} a(u, v)+b(u, u, v)+c(v, p) & =(f, v), & & \forall v \in V, \\
c(u, q) & =0, & & \forall q \in M,
\end{aligned}\right.
$$

where $(\cdot, \cdot)$ denotes the usual inner product on $\left(L^{2}(\Omega)\right)^{N}$ and

$$
\begin{aligned}
a(u, v) & =\int_{\Omega} \nabla u \cdot \nabla v \mathrm{~d} x \\
b(u, v, w) & =\frac{1}{2}\left(\sum_{i, j=1}^{N} \int_{\Omega} u_{i} \frac{\partial v_{j}}{\partial x_{i}} w_{j} \mathrm{~d} x-\sum_{i, j=1}^{N} \int_{\Omega} u_{i} \frac{\partial w_{j}}{\partial x_{i}} v_{j} \mathrm{~d} x\right) \\
c(v, p) & =-\int_{\Omega} p \operatorname{div} v \mathrm{~d} x
\end{aligned}
$$

It is known [19] that

$$
\left\{\begin{array}{l}
b(u, v, w)=-b(u, w, v), \quad b(u, v, v)=0, \quad \forall u, v, w \in V \\
|b(u, v, w)| \leq C_{0}\|u\|\|v\|\|w\|, \quad \forall u, v, w \in V
\end{array}\right.
$$

where $\|v\|=a(v, v)^{\frac{1}{2}}, \forall v \in V$. In this paper $C$ with or without subscript denotes a positive constant. 
Under the condition

$$
R_{e}<\sqrt{\frac{1}{C_{0}\|f\|_{-1}}}
$$

equation (2.1) has a unique solution [19]. Here, $\|\cdot\|_{-1}$ denotes the norm on the dual space $H^{-1}(\Omega)$.

We now consider some finite element subspaces of $V$ and $M$. Let $V_{h} \subset V$ and $M_{h} \subset L^{2}(\Omega)$ be a family of finite element subspaces of $V$ and $M$, respectively, which satisfy the following LBB (inf-sup) condition:

$$
\inf _{q \in M_{h} \cap L_{0}^{2}(\Omega)} \sup _{v \in V_{h}} \frac{c(v, q)}{\|v\|\|q\|_{0}} \geq \beta_{0}>0
$$

where $\beta_{0}$ is a constant independent of the mesh size $h$ and $\|\cdot\|_{0}$ is the $L^{2}(\Omega)$ norm.

The finite element approximation of $(2.2)$ can be written as:

$$
\left\{\begin{aligned}
\frac{1}{R_{e}} a\left(u_{h}, v\right)+b\left(u_{h}, u_{h}, v\right)+c\left(v, p_{h}\right) & =(f, v), & & \forall v \in V_{h}, \\
c\left(u_{h}, q\right) & =0, & & \forall q \in M_{h} \cap L_{0}^{2}(\Omega),
\end{aligned}\right.
$$

for some $u_{h} \in V_{h}, p_{h} \in M_{h} \cap L_{0}^{2}(\Omega)$.

It is known [19] that under the assumptions (2.4), (2.5), the finite element equations (2.6) have a unique solution which satisfies the following estimates

$$
\left\|u_{h}\right\| \leq R_{e}\|f\|_{-1}
$$

and

$$
\left\|p_{h}\right\|_{0} \leq \frac{1}{\beta_{0}}\|f\|_{-1}\left(2+C_{0} R_{e}^{2}\|f\|_{-1}\right)
$$

Remark 2.1. There exist numerous finite element spaces satisfying the above conditions, especially condition (2.5). Refer to $[4,8]$ for details.

Remark 2.2. To ensure uniqueness of the pressure in the finite element equations (2.6), we may assume that the pressure has a zero mean value.

We now describe a domain decomposition procedure. First decompose $\Omega$ into two nonoverlapping subdomains $\Omega_{1}$ and $\Omega_{2}$ such that $\bar{\Omega}_{1} \cup \bar{\Omega}_{2}=\bar{\Omega}$ and $\Omega_{2}$ is not an interior subdomain (i.e., $\bar{\Omega}_{2} \not \subset \Omega$ ). We suppose that the interface $\Gamma=\bar{\Omega}_{1} \cap \bar{\Omega}_{2}$ does not cut across any finite element. We also assume that the point mentioned in Remark 2.2 belongs to $\Omega_{1}$. Define, for $k=1,2$,

$$
\begin{aligned}
V_{h, k} & =\left\{\left.v_{h}\right|_{\Omega_{k}}: v_{h} \in V_{h}\right\}, \\
V_{h, k}^{0} & =V_{h, k} \cap\left(H_{0}^{1}\left(\Omega_{k}\right)\right)^{N}, \\
M_{h, k} & =\left\{\left.q_{h}\right|_{\Omega_{k}}: q_{h} \in M_{h}\right\}, \\
\Phi_{h} & =\left\{\left.v_{h}\right|_{\Gamma}: v_{h} \in V_{h}\right\} .
\end{aligned}
$$


We now consider the following two-domain problem: find $\left(u_{h, k}, p_{h, k}\right) \in V_{h, k} \times M_{h, k}, k=1,2$ such that

$$
\begin{aligned}
\frac{1}{R_{e}} a_{1}\left(u_{h, 1}, v\right)+b_{1}\left(u_{h, 1}, u_{h, 1}, v\right)+c_{1}\left(v, p_{h, 1}\right)= & (f, v)_{1}, \quad \forall v \in V_{h, 1}^{0}, \\
c_{1}\left(u_{h, 1}, q\right)= & 0, \quad \forall q \in M_{h, 1} \cap L_{0}^{2}\left(\Omega_{1}\right), \\
\gamma_{0} u_{h, 1}= & \gamma_{0} u_{h, 2}, \quad \text { on } \Gamma, \\
\frac{1}{R_{e}} a_{2}\left(u_{h, 2}, v\right)+b_{2}\left(u_{h, 2}, u_{h, 2}, v\right)+c_{2}\left(v, p_{h, 2}\right)= & (f, v)_{2}+\left(f, \rho_{h, 1} \gamma_{0} v\right)_{1} \\
& -\frac{1}{R_{e}} a_{1}\left(u_{h, 1}, \rho_{h, 1} \gamma_{0} v\right) \\
& -b_{1}\left(u_{h, 1}, u_{h, 1}, \rho_{h, 1} \gamma_{0} v\right) \\
& -c_{1}\left(\rho_{h, 1} \gamma_{0} v, p_{h, 1}\right), \quad \forall v \in V_{h, 2}, \\
c_{2}\left(u_{h, 2}, q\right)= & 0, \quad \forall q \in M_{h, 2} \\
& \int_{\Omega_{1}} p_{h, 1} \mathrm{~d} x=-\int_{\Omega_{2}} p_{h, 2} \mathrm{~d} x .
\end{aligned}
$$

Here, $(\cdot, \cdot)_{k}$ denotes the inner product on $\left(L^{2}\left(\Omega_{k}\right)\right)^{N}, \gamma_{0} v \in \Phi_{h}$ is the trace on $\Gamma$ of the function $v \in V_{h}$ and $\rho_{h, k}(k=1,2)$ is an extension operator from $\Phi_{h}$ to $V_{h, k}$. In practical implementations, these extensions can be taken equal to the finite element interpolant which belongs to $V_{h, i}$, equal to $\gamma_{0} v$ at the nodes on the interface, and vanishes at the internal nodes in $\Omega_{i}$. For $k=1,2$, let

$$
\begin{aligned}
a_{k}(u, v) & =\int_{\Omega_{k}} \nabla u \cdot \nabla v \mathrm{~d} x, \quad \forall u, v \in V_{h, k} \\
b_{k}(u, v, w) & =\frac{1}{2}\left(\sum_{i, j=1}^{N} \int_{\Omega_{k}} u_{i} \frac{\partial v_{j}}{\partial x_{i}} w_{j} \mathrm{~d} x-\sum_{i, j=1}^{N} \int_{\Omega_{k}} u_{i} \frac{\partial w_{j}}{\partial x_{i}} v_{j} \mathrm{~d} x\right), \forall u, v, w \in V_{h, k}, \\
c_{k}(v, p) & =-\int_{\Omega_{k}} p \operatorname{div} v \mathrm{~d} x, \quad \forall v \in V_{h, k}, p \in M_{h, k} .
\end{aligned}
$$

Define

Then we have [19]

$$
\|v\|_{k}=a_{k}(v, v)^{\frac{1}{2}}, \quad \forall v \in V_{h, k} .
$$

$$
\left\{\begin{array}{l}
b_{k}(u, v, w)=-b_{k}(u, w, v), \quad b_{k}(u, v, v)=0, \quad \forall u, v, w \in V_{h, k} \\
\left|b_{k}(u, v, w)\right| \leq C\|u\|_{k}\|v\|_{k}\|w\|_{k}, \quad \forall u, v, w \in V_{h, k}
\end{array}\right.
$$

Lemma 2.1. The function $\left(u_{h}, p_{h}\right)$ is the solution of (2.6) if and only if $\left(u_{h, 1}, p_{h, 1}\right)$ and $\left(u_{h, 2}, p_{h, 2}\right)$ satisfy the split problems (2.9)-(2.14), where $u_{h, k}=\left.u_{h}\right|_{\Omega_{k}}, p_{h, k}=\left.p_{h}\right|_{\Omega_{k}}, k=1,2$.

Proof. Let us first check that if $\left(u_{h}, p_{h}\right)$ is the solution of $(2.6)$, then $\left(u_{h, 1}, p_{h, 1}\right)$ and $\left(u_{h, 2}, p_{h, 2}\right)$ satisfy $(2.9)-(2.14)$.

For any $v \in V_{h, 1}^{0}, q \in M_{h, 1} \cap L_{0}^{2}\left(\Omega_{1}\right)$, define $(\tilde{v}, \tilde{q})$ as follows,

$$
\begin{array}{ll}
\tilde{v}=v \text { in } \Omega_{1}, & \tilde{v}=0 \text { in } \Omega_{2} \\
\tilde{q}=q \text { in } \Omega_{1}, & \tilde{q}=0 \text { in } \Omega_{2} .
\end{array}
$$

Then

$$
\frac{1}{R_{e}} a_{1}\left(u_{h, 1}, v\right)+b_{1}\left(u_{h, 1}, u_{h, 1}, v\right)+c_{1}\left(v, p_{h, 1}\right)=\frac{1}{R_{e}} a\left(u_{h}, \tilde{v}\right)+b\left(u_{h}, u_{h}, \tilde{v}\right)+c\left(\tilde{v}, p_{h}\right)=(f, \tilde{v})=(f, v)_{1},
$$


and

$$
c_{1}\left(u_{h, 1}, q\right)=c\left(u_{h}, \tilde{q}\right)=0,
$$

which are (2.9), (2.10). Equations (2.11), (2.14) are obviously satisfied because $u_{h} \in V_{h}$, and $p_{h} \in M_{h} \cap L_{0}^{2}(\Omega)$.

On the other hand, for any $v \in V_{h, 2}$, define $\tilde{v} \in V_{h}$ as follows,

$$
\tilde{v}=\rho_{h, 1} \gamma_{0} v \text { in } \Omega_{1}, \quad \tilde{v}=v \text { in } \Omega_{2} .
$$

Then for any $v \in V_{h, 2}, q \in M_{h, 2}$, we have

$$
\begin{aligned}
& \frac{1}{R_{e}} a_{2}\left(u_{h, 2}, v\right)+b_{2}\left(u_{h, 2}, u_{h, 2}, v\right)+c_{2}\left(v, p_{h, 2}\right) \\
= & \frac{1}{R_{e}} a\left(u_{h}, \tilde{v}\right)+b\left(u_{h}, u_{h}, \tilde{v}\right)+c\left(\tilde{v}, p_{h}\right) \\
& -\frac{1}{R_{e}} a_{1}\left(u_{h, 1}, \rho_{h, 1} \gamma_{0} v\right)-b_{1}\left(u_{h, 1}, u_{h, 1}, \rho_{h, 1} \gamma_{0} v\right)-c_{1}\left(\rho_{h, 1} \gamma_{0} v, p_{h, 1}\right) \\
= & (f, v)_{2}+\left(f, \rho_{h, 1} \gamma_{0} v\right)_{1} \\
& -\frac{1}{R_{e}} a_{1}\left(u_{h, 1}, \rho_{h, 1} \gamma_{0} v\right)-b_{1}\left(u_{h, 1}, u_{h, 1}, \rho_{h, 1} \gamma_{0} v\right)-c_{1}\left(\rho_{h, 1} \gamma_{0} v, p_{h, 1}\right),
\end{aligned}
$$

and

where $\tilde{q}$ is defined by

$$
c_{2}\left(u_{h, 2}, q\right)=c\left(u_{h}, \tilde{q}\right)
$$

$\tilde{q}=0$ in $\Omega_{1}, \quad \tilde{q}=q$ in $\Omega_{2}$.

Although $\tilde{q} \notin L_{0}^{2}(\Omega)$, we still have $c_{2}\left(u_{h}, \tilde{q}\right)=0$, because

$$
c\left(u_{h}, q\right)=0, \quad \forall q \in M_{h},
$$

here $M_{h}$ is the finite element space which is no the restriction $\int_{\Omega} q_{h} \mathrm{~d} x=0$ ( $c f$. [17] for details).

Conversely, suppose $\left(u_{h, 1}, p_{h, 1}\right)$ and $\left(u_{h, 2}, p_{h, 2}\right)$ are the solutions of $(2.9)-(2.13)$. Define $\left(u_{h}, p_{h}\right)$ as follows:

$$
\begin{aligned}
& u_{h}=u_{h, 1} \text { in } \Omega_{1}, \quad u_{h}=u_{h, 2} \text { in } \Omega_{2} ; \\
& p_{h}=p_{h, 1} \text { in } \Omega_{1}, \quad p_{h}=p_{h, 2} \text { in } \Omega_{2} .
\end{aligned}
$$

We now prove that $\left(u_{h}, p_{h}\right)$ is the solution of (2.6). In fact, for any $v_{h} \in V_{h}$, define $v_{h, k}=\left.v\right|_{\Omega_{k}}(k=1,2)$ and $v_{h, 1}^{0}=v_{h, 1}-\rho_{h, 1} \gamma_{0} v_{h, 1}=v_{h, 1}-\rho_{h, 1} \gamma_{0} v_{h, 2}$. Then

$$
\begin{aligned}
& \frac{1}{R_{e}} a\left(u_{h}, v_{h}\right)+b\left(u_{h}, u_{h}, v_{h}\right)+c\left(v_{h}, p_{h}\right)=\frac{1}{R_{e}} a_{1}\left(u_{h, 1}, v_{h, 1}\right)+b_{1}\left(u_{h, 1}, u_{h, 1}, v_{h, 1}\right)+c_{1}\left(v_{h, 1}, p_{h, 1}\right) \\
& +\frac{1}{R_{e}} a_{2}\left(u_{h, 2}, v_{h, 2}\right)+b_{2}\left(u_{h, 2}, u_{h, 2}, v_{h, 2}\right)+c_{2}\left(v_{h, 2}, p_{h, 2}\right) \\
= & \frac{1}{R_{e}} a_{1}\left(u_{h, 1}, v_{h, 1}^{0}\right)+b_{1}\left(u_{h, 1}, u_{h, 1}, v_{h, 1}^{0}\right)+c_{1}\left(v_{h, 1}^{0}, p_{h, 1}\right) \\
& +\frac{1}{R_{e}} a_{1}\left(u_{h, 1}, \rho_{h, 1} \gamma_{0} v_{h, 2}\right)+b_{1}\left(u_{h, 1}, u_{h, 1}, \rho_{h, 1} \gamma_{0} v_{h, 2}\right)+c_{1}\left(\rho_{h, 1} \gamma_{0} v_{h, 2}, p_{h, 1}\right) \\
& +\frac{1}{R_{e}} a_{2}\left(u_{h, 2}, v_{h, 2}\right)+b_{2}\left(u_{h, 2}, u_{h, 2}, v_{h, 2}\right)+c_{2}\left(v_{h, 2}, p_{h, 2}\right) \\
= & \left(f, v_{h, 1}^{0}\right)_{1}+\left(f, \rho_{h, 1} \gamma_{0} v_{h, 2}\right)_{1}+\left(f, v_{h, 2}\right)_{2} \\
= & \left(f, v_{h}\right) .
\end{aligned}
$$


On the other hand, for any $q_{h}=q_{h, 1}+q_{h, 2} \in M_{h}$ with $q_{h, k} \in M_{h, k}$, using the same argument as the Lemma 5.3 .9 in [17], we can get

$$
c\left(u_{h}, q_{h}\right)=c_{1}\left(u_{h, 1}, q_{h, 1}\right)+c_{2}\left(u_{h, 2}, q_{h, 2}\right)=0 .
$$

We have proved Lemma 2.1.

\section{The Dirichlet-Neumann iterative algorithm}

In order to define our DDM algorithm, we first analyze a property of the trace on $\Gamma$ of the discrete solution $u_{h}$. Note

$$
\left.\int_{\Gamma} u_{h}\right|_{\Gamma} \cdot n \mathrm{~d} s=\left.\int_{\partial \Omega_{2}} u_{h}\right|_{\Gamma} \cdot n \mathrm{~d} s=\int_{\Omega_{2}} \nabla \cdot u_{h} \mathrm{~d} x=0
$$

where $n$ denotes the unit normal vector of the boundary $\partial \Omega_{2}$. Therefore, we introduce the following trace space

$$
\hat{\Phi}_{h}=\left\{\eta_{h} \in \Phi_{h} \mid \int_{\Gamma} \eta_{h} \cdot n \mathrm{~d} s=0\right\} .
$$

We now propose our two-subdomain decomposition method to solve equation (2.6).

For any $g_{h}^{0} \in \hat{\Phi}_{h}$, define the two sequences $\left(u_{h, 1}^{n}, p_{h, 1}^{n}\right) \in V_{h, 1} \times M_{h, 1}$ and $\left(u_{h, 2}^{n}, p_{h, 2}^{n}\right) \in V_{h, 2} \times M_{h, 2}$ as follows:

$$
\left\{\begin{aligned}
\frac{1}{R_{e}} a_{1}\left(u_{h, 1}^{n}, v\right)+b_{1}\left(u_{h, 1}^{n-1}, u_{h, 1}^{n}, v\right)+c_{1}\left(v, p_{h, 1}^{n}\right) & =(f, v)_{1}, \quad \forall v \in V_{h, 1}^{0} \\
c_{1}\left(u_{h, 1}^{n}, q\right) & =0, \quad \forall q \in M_{h, 1} \cap L_{0}^{2}\left(\Omega_{1}\right) \\
\gamma_{0} u_{h, 1}^{n} & =g_{h}^{n-1}, \quad \text { on } \Gamma, \\
\int_{\Omega_{1}} p_{h, 1}^{n} \mathrm{~d} x & =-\int_{\Omega_{2}} p_{h, 2}^{n-1} \mathrm{~d} x,
\end{aligned}\right.
$$

and

$$
\left\{\begin{aligned}
& \frac{1}{R_{e}} a_{2}\left(u_{h, 2}^{n}, v\right)+b_{2}\left(u_{h, 2}^{n-1}, u_{h, 2}^{n}, v\right)+c_{2}\left(v, p_{h, 2}^{n}\right)=(f, v)_{2}+\left(f, \rho_{h, 1} \gamma_{0} v\right)_{1} \\
&-\frac{1}{R_{e}} a_{1}\left(u_{h, 1}^{n}, \rho_{h, 1} \gamma_{0} v\right) \\
&-b_{1}\left(u_{h, 1}^{n-1}, u_{h, 1}^{n}, \rho_{h, 1} \gamma_{0} v\right) \\
&-c_{1}\left(\rho_{h, 1} \gamma_{0} v, p_{h, 1}^{n}\right), \quad \forall v \in V_{h, 2} \\
& c_{2}\left(u_{h, 2}^{n}, q\right)=0, \quad \forall q \in M_{h, 2},
\end{aligned}\right.
$$

where we have set

$$
g_{h}^{n}=\theta_{n} \gamma_{0} u_{h, 2}^{n}+\left(1-\theta_{n}\right) g_{h}^{n-1},
$$

where $\theta_{n}<1$ are positive numbers to be fixed later on.

Remark 3.1. For each $n \geq 1,(3.1)$ and (3.2) are finite element approximations to the N-S equations with nonhomogeneous boundary conditions. In this case, the inf-sup condition (2.5) is still satisfied on each subdomain. This ensures that both subproblems (3.1) and (3.2) have a unique solution.

We now consider the convergence rate of the iterative algorithm (3.1)-(3.2). The main theorem is that the iterations converge to the exact solution geometrically, with the rate independent of $h$. We shall develop six lemmas before stating and proving the main theorem. These lemmas contain the basic estimates which are needed in the proof of the main theorem. From (3.1)-(3.2), we can derive the following error equations for $\xi_{h, k}^{n}=u_{h, k}^{n}-u_{h, k} \in V_{h, k}$ and $\eta_{h, k}^{n}=p_{h, k}^{n}-p_{h, k} \in M_{h, k}$.

$$
\left\{\begin{aligned}
\frac{1}{R_{e}} a_{1}\left(\xi_{h, 1}^{n}, v\right)+c_{1}\left(v, \eta_{h, 1}^{n}\right) & =-b_{1}\left(u_{h, 1}^{n-1}, u_{h, 1}^{n}, v\right)+b_{1}\left(u_{h, 1}, u_{h, 1}, v\right), \quad \forall v \in V_{h, 1}^{0} \\
c_{1}\left(\xi_{h, 1}^{n}, q\right) & =0, \quad \forall q \in M_{h, 1} \cap L_{0}^{2}(\Omega) \\
\gamma_{0} \xi_{h, 1}^{n} & =g_{h}^{n-1}-\left.u_{h, 2}\right|_{\Gamma}, \quad \text { on } \Gamma \\
\int_{\Omega_{1}} \eta_{h, 1}^{n} \mathrm{~d} x & =-\int_{\Omega_{2}} \eta_{h, 2}^{n-1} \mathrm{~d} x
\end{aligned}\right.
$$


and

$$
\left\{\begin{aligned}
\frac{1}{R_{e}} a_{2}\left(\xi_{h, 2}^{n}, v\right)+c_{2}\left(v, \eta_{h, 2}^{n}\right)= & -b_{2}\left(u_{h, 2}^{n-1}, u_{h, 2}^{n}, v\right)+b_{2}\left(u_{h, 2}, u_{h, 2}, v\right) \\
& -\frac{1}{R_{e}} a_{1}\left(\xi_{h, 1}^{n}, \rho_{h, 1} \gamma_{0} v\right)-c_{1}\left(\rho_{h, 1} \gamma_{0} v, \eta_{h, 1}^{n}\right) \\
& -b_{1}\left(u_{h, 1}^{n-1}, u_{h, 1}^{n}, \rho_{h, 1} \gamma_{0} v\right)+b_{1}\left(u_{h, 1}, u_{h, 1}, \rho_{h, 1} \gamma_{0} v\right), \quad \forall v \in V_{h, 2} \\
c_{2}\left(\xi_{h, 2}^{n}, q\right)= & 0, \quad \forall q \in M_{h, 2}
\end{aligned}\right.
$$

In addition, we have

$$
\gamma_{0} \xi_{h, 1}^{n+1}=\theta_{n} \gamma_{0} \xi_{h, 2}^{n}+\left(1-\theta_{n}\right) \gamma_{0} \xi_{h, 1}^{n}
$$

Problem (3.3) can be further split into two subproblems: find $\xi_{h, 1_{*}}^{n} \in V_{h, 1}, \eta_{h, 1_{*}}^{n} \in M_{h, 1}$ such that

$$
\left\{\begin{aligned}
\frac{1}{R_{e}} a_{1}\left(\xi_{h, 1_{*}}^{n}, v\right)+c_{1}\left(v, \eta_{h, 1_{*}}^{n}\right) & =0, \quad \forall v \in V_{h, 1}^{0} \\
c_{1}\left(\xi_{h, 1_{*}}^{n}, q\right) & =0, \quad \forall q \in M_{h, 1} \cap L_{0}^{2}\left(\Omega_{1}\right) \\
\gamma_{0} \xi_{h, 1_{*}}^{n} & =\gamma_{0} \xi_{h, 1}^{n}, \quad \text { on } \Gamma, \\
\int_{\Omega_{1}} \eta_{h, 1}^{n} \mathrm{~d} x & =-\int_{\Omega_{2}} \eta_{h, 2}^{n-1} \mathrm{~d} x
\end{aligned}\right.
$$

and find $\xi_{h, 1_{* *}}^{n} \in V_{h, 1}^{0}, \eta_{h, 1_{* *}}^{n} \in M_{h, 1} \cap L_{0}^{2}\left(\Omega_{1}\right)$ such that

$$
\left\{\begin{aligned}
\frac{1}{R_{e}} a_{1}\left(\xi_{h, 1_{* *}}^{n}, v\right)+c_{1}\left(v, \eta_{h, 1_{* *}}^{n}\right) & =-b_{1}\left(u_{h, 1}^{n-1}, u_{h, 1}^{n}, v\right)+b_{1}\left(u_{h, 1}, u_{h, 1}, v\right), \quad \forall v \in V_{h, 1}^{0} \\
c_{1}\left(\xi_{h, 1_{* *}}^{n}, q\right) & =0, \quad \forall q \in M_{h, 1} \cap L_{0}^{2}\left(\Omega_{1}\right) \\
\gamma_{0} \xi_{h, 1_{* *}}^{n} & =0, \quad \text { on } \Gamma .
\end{aligned}\right.
$$

It is easy to see that

$$
\left\{\begin{array}{l}
\xi_{h, 1}^{n}=\xi_{h, 1_{*}}^{n}+\xi_{h, 1_{* *}}^{n} \\
\eta_{h, 1}^{n}=\eta_{h, 1_{*}}^{n}+\eta_{h, 1_{* *}}^{n}
\end{array} .\right.
$$

We are now ready to state and prove the first lemma.

\section{Lemma 3.1.}

$$
\begin{aligned}
& \text { (1) }\left\|\xi_{h, 1}^{n}\right\|_{1}^{2}=\left\|\xi_{h, 1_{*}}^{n}\right\|_{1}^{2}+\left\|\xi_{h, 1_{* *}}^{n}\right\|_{1}^{2}, \\
& \text { (2) }\left\|\xi_{h, 1_{* *}}^{n}\right\|_{1} \leq C R_{e} G_{1}^{n}
\end{aligned}
$$

where $G_{1}^{n} \hat{=}\left(\left\|\xi_{h, 1}^{n-1}\right\|_{1}+\left\|u_{h, 1}\right\|_{1}\right)\left\|\xi_{h, 1}^{n}\right\|_{1}+\left\|u_{h, 1}\right\|_{1}\left\|\xi_{h, 1}^{n-1}\right\|_{1}$.

Proof. Because $\xi_{h, 1_{* *}}^{n} \in V_{h, 1}^{0}$, we take $v=\xi_{h, 1_{* *}}^{n}$ in (3.6) and obtain

$$
\frac{1}{R_{e}} a_{1}\left(\xi_{h, 1_{*}}^{n}, \xi_{h, 1_{* *}}^{n}\right)+c_{1}\left(\xi_{h, 1_{* *}}^{n}, \eta_{h, 1_{*}}^{n}\right)=0
$$

Using the fact

we get

$$
c_{1}\left(\xi_{h, 1_{* *}}^{n}, \eta_{h, 1_{*}}^{n}\right)=0
$$

$$
a_{1}\left(\xi_{h, 1_{*}}^{n}, \xi_{h, 1_{* *}}^{n}\right)=0
$$

Finally,

$$
\begin{aligned}
\left\|\xi_{h, 1}^{n}\right\|_{1}^{2} & =\left\|\xi_{h, 1_{*}}^{n}+\xi_{h, 1_{* *}}^{n}\right\|_{1}^{2} \\
& =\left\|\xi_{h, 1_{*}}^{n}\right\|_{1}^{2}+\left\|\xi_{h, 1_{* *}}^{n}\right\|_{1}^{2}+2 a_{1}\left(\xi_{h, 1_{*}}^{n}, \xi_{h, 1_{* *}}^{n}\right) \\
& =\left\|\xi_{h, 1_{*}}^{n}\right\|_{1}^{2}+\left\|\xi_{h, 1_{* *}}^{n}\right\|_{1}^{2}
\end{aligned}
$$

which is equation (1) in Lemma 3.1. 
We now prove (2) of Lemma 3.1. Taking $v=\xi_{h, 1_{* *}}^{n}$ in (3.7), we have

$$
\begin{aligned}
\frac{1}{R_{e}} a_{1}\left(\xi_{h, 1_{* *}}^{n}, \xi_{h, 1_{* *}}^{n}\right)= & -b_{1}\left(u_{h, 1}^{n-1}, u_{h, 1}^{n}, \xi_{h, 1_{* *}}^{n}\right)+b_{1}\left(u_{h, 1}, u_{h, 1}, \xi_{h, 1_{* *}}^{n}\right) \\
= & -b_{1}\left(\xi_{h, 1}^{n-1}, \xi_{h, 1}^{n}, \xi_{h, 1_{* *}}^{n}\right)-b_{1}\left(\xi_{h, 1}^{n-1}, u_{h, 1}, \xi_{h, 1_{* *}}^{n}\right) \\
& -b_{1}\left(u_{h, 1}, \xi_{h, 1}^{n}, \xi_{h, 1_{* *}}^{n}\right) .
\end{aligned}
$$

Then we have

$$
\left\|\xi_{h, 1_{* *}}^{n}\right\|_{1}^{2} \leq C R_{e}\left[\left(\left\|\xi_{h, 1}^{n-1}\right\|_{1}+\left\|u_{h, 1}\right\|_{1}\right)\left\|\xi_{h, 1}^{n}\right\|_{1}+\left\|u_{h, 1}\right\|_{1}\left\|\xi_{h, 1}^{n-1}\right\|_{1}\right],
$$

which implies (2) of Lemma 3.1.

Next we introduce two Stokes extension operators which were first proposed in [16]. For any $\psi \in \hat{\Phi}_{h}$, define $\left(E_{h, k} \psi, P_{h, k} \psi\right) \in V_{h, k} \times M_{h, k}$ as follows:

$$
\left\{\begin{aligned}
\frac{1}{R_{e}} a_{k}\left(E_{h, k} \psi, v\right)+c_{k}\left(v, P_{h, k} \psi\right) & =0, \quad \forall v \in V_{h, k}^{0}, \\
c_{k}\left(E_{h, k} \psi, q\right) & =0, \quad \forall q \in M_{h, k}, \\
\gamma_{0} E_{h, k} \psi & =\psi
\end{aligned}\right.
$$

Remark 3.2. To ensure that the above Stokes subproblem on $\Omega_{2}$ has a unique solution, we assume that the function $q \in M_{h, 2}$ satisfies $\bar{q}=0$, where

$$
\bar{q} \hat{=} \frac{1}{\left|\Omega_{2}\right|} \int_{\Omega_{2}} q \mathrm{~d} x .
$$

Note that for any $q \in M_{h, 2}$ which does not satisfy the above property, we still have

$$
c_{2}\left(E_{h, 2} \psi, q\right)=c_{2}\left(E_{h, 2} \psi, q-\bar{q}\right)+c_{2}\left(E_{h, 2} \psi, \bar{q}\right)=-\bar{q} \int_{\Gamma} \psi \cdot n \mathrm{~d} s=0,
$$

as $\psi \in \hat{\Phi}_{h}$.

Lemma 3.2. [16] For any $\psi \in \hat{\Phi}_{h}$,

$$
\begin{aligned}
& \text { (1) } a_{1}\left(E_{h, 1} \psi, E_{h, 1} \psi\right) \leq \sigma a_{2}\left(E_{h, 2} \psi, E_{h, 2} \psi\right), \\
& \text { (2) } a_{2}\left(E_{h, 2} \psi, E_{h, 2} \psi\right) \leq \tau a_{1}\left(E_{h, 1} \psi, E_{h, 1} \psi\right),
\end{aligned}
$$

where $\sigma, \tau$ are constants independent of the mesh size $h$.

The next lemma shows that the algorithm is independent of the choice of the extension operator $\rho_{h, k}$. While the trivial extension may be more convenient in practice, $E_{h, k}$ may be more convenient theoretically. Both choices lead to the same algorithm.

Lemma 3.3. The first formula in (3.4) is equivalent to

$$
\begin{aligned}
\frac{1}{R_{e}} a_{2}\left(\xi_{h, 2}^{n}, v\right)+c_{2}\left(v, \eta_{h, 2}^{n}\right)= & -b_{2}\left(u_{h, 2}^{n-1}, u_{h, 2}^{n}, v\right)+b_{2}\left(u_{h, 2}, u_{h, 2}, v\right) \\
& -\frac{1}{R_{e}} a_{1}\left(\xi_{h, 1}^{n}, E_{h, 1} \gamma_{0} v\right)-c_{1}\left(E_{h, 1} \gamma_{0} v, \eta_{h, 1}^{n}\right) \\
& -b_{1}\left(u_{h, 1}^{n-1}, u_{h, 1}^{n}, E_{h, 1} \gamma_{0} v\right)+b_{1}\left(u_{h, 1}, u_{h, 1}, E_{h, 1} \gamma_{0} v\right), \quad \forall v \in V_{h, 2} .
\end{aligned}
$$


Proof. For any $v \in V_{h, 2}$, because $E_{h, 1} \gamma_{0} v-\rho_{h, 1} \gamma_{0} v \in V_{h, 1}^{0}$, we have, by (3.3),

$$
\begin{aligned}
0= & \frac{1}{R_{e}} a_{1}\left(\xi_{h, 1}^{n}, E_{h, 1} \gamma_{0} v-\rho_{h, 1} \gamma_{0} v\right)+b_{1}\left(u_{h, 1}^{n-1}, u_{h, 1}^{n}, E_{h, 1} \gamma_{0} v-\rho_{h, 1} \gamma_{0} v\right) \\
& -b_{1}\left(u_{h, 1}, u_{h, 1}, E_{h, 1} \gamma_{0} v-\rho_{h, 1} \gamma_{0} v\right)+c_{1}\left(E_{h, 1} \gamma_{0} v-\rho_{h, 1} \gamma_{0} v, \eta_{h, 1}^{n}\right) .
\end{aligned}
$$

That is,

$$
\begin{aligned}
\frac{1}{R_{e}} a_{1}\left(\xi_{h, 1}^{n}, \rho_{h, 1} \gamma_{0} v\right)+b_{1}\left(u_{h, 1}^{n-1}, u_{h, 1}^{n}, \rho_{h, 1} \gamma_{0} v\right) & \\
& -b_{1}\left(u_{h, 1}, u_{h, 1}, \rho_{h, 1} \gamma_{0} v\right)+c_{1}\left(\rho_{h, 1} \gamma_{0} v, \eta_{h, 1}^{n}\right) \\
= & \frac{1}{R_{e}} a_{1}\left(\xi_{h, 1}^{n}, E_{h, 1} \gamma_{0} v\right)+b_{1}\left(u_{h, 1}^{n-1}, u_{h, 1}^{n}, E_{h, 1} \gamma_{0} v\right) \\
& -b_{1}\left(u_{h, 1}, u_{h, 1}, E_{h, 1} \gamma_{0} v\right)+c_{1}\left(E_{h, 1} \gamma_{0} v, \eta_{h, 1}^{n}\right)
\end{aligned}
$$

which, together with (3.4), yields Lemma 3.3.

\section{Lemma 3.4.}

$$
\left\|E_{h, 2} \gamma_{0} \xi_{h, 2}^{n}\right\|_{2} \leq\left\|\xi_{h, 2}^{n}\right\|_{2}+C R_{e} G_{2}^{n}
$$

where $G_{2}^{n}=\left(\left\|\xi_{h, 2}^{n-1}\right\|_{2}+\left\|u_{h, 2}\right\|_{2}\right)\left\|\xi_{h, 2}^{n}\right\|_{2}+\left\|u_{h, 2}\right\|_{2}\left\|\xi_{h, 2}^{n-1}\right\|_{2}$.

Proof. Taking $v \in V_{h, 2}^{0}$ in (3.4), we get

$$
\frac{1}{R_{e}} a_{2}\left(\xi_{h, 2}^{n}, v\right)+c_{2}\left(v, \eta_{h, 2}^{n}\right)=-b_{2}\left(u_{h, 2}^{n-1}, u_{h, 2}^{n}, v\right)+b_{2}\left(u_{h, 2}, u_{h, 2}, v\right)
$$

On the other hand, based on the definition of the extension operators $E_{h, 2}, P_{h, 2}$, we have

$$
\frac{1}{R_{e}} a_{2}\left(E_{h, 2} \gamma_{0} \xi_{h, 2}^{n}, v\right)+c_{2}\left(v, P_{h, 2} \gamma_{0} \xi_{h, 2}^{n}\right)=0, \quad \forall v \in V_{h, 2}^{0}
$$

Combining the above two equalities, we have

$$
\frac{1}{R_{e}} a_{2}\left(\xi_{h, 2}^{n}-E_{h, 2} \gamma_{0} \xi_{h, 2}^{n}, v\right)+c_{2}\left(v, \eta_{h, 2}^{n}-P_{h, 2} \gamma_{0} \xi_{h, 2}^{n}\right)=-b_{2}\left(u_{h, 2}^{n-1}, u_{h, 2}^{n}, v\right)+b_{2}\left(u_{h, 2}, u_{h, 2}, v\right) .
$$

Taking $v=\xi_{h, 2}^{n}-E_{h, 2} \gamma_{0} \xi_{h, 2}^{n}$ in the above inequality, and noting the fact

$$
c_{2}\left(\xi_{h, 2}^{n}-E_{h, 2} \gamma_{0} \xi_{h, 2}^{n}, q\right)=0, \quad \forall q \in M_{h, 2},
$$

we have

$$
\begin{aligned}
\left\|\xi_{h, 2}^{n}-E_{h, 2} \gamma_{0} \xi_{h, 2}^{n}\right\|_{2}^{2}= & -R_{e} b_{2}\left(u_{h, 2}^{n-1}, u_{h, 2}^{n}, \xi_{h, 2}^{n}-E_{h, 2} \gamma_{0} \xi_{h, 2}^{n}\right)+R_{e} b_{2}\left(u_{h, 2}, u_{h, 2}, \xi_{h, 2}^{n}-E_{h, 2} \gamma_{0} \xi_{h, 2}^{n}\right) \\
= & -R_{e} b_{2}\left(\xi_{h, 2}^{n-1}, \xi_{h, 2}^{n}, \xi_{h, 2}^{n}-E_{h, 2} \gamma_{0} \xi_{h, 2}^{n}\right)-R_{e} b_{2}\left(\xi_{h, 2}^{n-1}, u_{h, 2}, \xi_{h, 2}^{n}-E_{h, 2} \gamma_{0} \xi_{h, 2}^{n}\right) \\
& -R_{e} b_{2}\left(u_{h, 2}, \xi_{h, 2}^{n}, \xi_{h, 2}^{n}-E_{h, 2} \gamma_{0} \xi_{h, 2}^{n}\right) \\
& \leq C R_{e}\left[\left(\left\|\xi_{h, 2}^{n-1}\right\|_{2}+\left\|u_{h, 2}\right\|_{2}\right)\left\|\xi_{h, 2}^{n}\right\|_{2}+\left\|u_{h, 2}\right\|_{2}\left\|\xi_{h, 2}^{n-1}\right\|_{2}\right]\left\|\xi_{h, 2}^{n}-E_{h, 2} \gamma_{0} \xi_{h, 2}^{n}\right\|_{2}
\end{aligned}
$$

Then

$$
\left\|\xi_{h, 2}^{n}-E_{h, 2} \gamma_{0} \xi_{h, 2}^{n}\right\|_{2} \leq C R_{e}\left(\left\|\xi_{h, 2}^{n-1}\right\|_{2}+\left\|u_{h, 2}\right\|_{2}\right)\left\|\xi_{h, 2}^{n}\right\|_{2}+\left\|u_{h, 2}\right\|_{2}\left\|\xi_{h, 2}^{n-1}\right\|_{2}
$$


which, together with the triangle inequality, yields

$$
\begin{aligned}
\left\|E_{h, 2} \gamma_{0} \xi_{h, 2}^{n}\right\|_{2} & \leq\left\|\xi_{h, 2}^{n}\right\|_{2}+\left\|\xi_{h, 2}^{n}-E_{h, 2} \gamma_{0} \xi_{h, 2}^{n}\right\|_{2} \\
& \leq\left\|\xi_{h, 2}^{n}\right\|_{2}+C R_{e}\left[\left(\left\|\xi_{h, 2}^{n-1}\right\|_{2}+\left\|u_{h, 2}\right\|_{2}\right)\left\|\xi_{h, 2}^{n}\right\|_{2}+\left\|u_{h, 2}\right\|_{2}\left\|\xi_{h, 2}^{n-1}\right\|_{2}\right] .
\end{aligned}
$$

This completes the proof of Lemma 3.4.

Lemma 3.5. Let $\varepsilon=C R_{e}$, then

$$
-\left\|\xi_{h, 2}^{n}\right\|_{2}^{2} \leq-\frac{1}{3 \tau}\left\|\xi_{h, 1}^{n}\right\|_{1}^{2}+\varepsilon^{2}\left(G_{2}^{n}\right)^{2}+\frac{4}{\tau} \varepsilon^{2}\left(G_{1}^{n}\right)^{2} .
$$

Proof. Taking $v=E_{h, 2} \gamma_{0} \xi_{h, 1}^{n}$ in (3.9), we have

$$
\begin{aligned}
\frac{1}{R_{e}} a_{2}\left(\xi_{h, 2}^{n}, E_{h, 2} \gamma_{0} \xi_{h, 1}^{n}\right)= & -b_{2}\left(u_{h, 2}^{n-1}, u_{h, 2}^{n}, E_{h, 2} \gamma_{0} \xi_{h, 1}^{n}\right)+b_{2}\left(u_{h, 2}, u_{h, 2}, E_{h, 2} \gamma_{0} \xi_{h, 1}^{n}\right)-\frac{1}{R_{e}} a_{1}\left(\xi_{h, 1}^{n}, \xi_{h, 1_{*}}^{n}\right) \\
& -b_{1}\left(u_{h, 1}^{n-1}, u_{h, 1}^{n}, \xi_{h, 1_{*}}^{n}\right)+b_{1}\left(u_{h, 1}, u_{h, 1}, \xi_{h, 1_{*}}^{n}\right) .
\end{aligned}
$$

In the above, we have used the fact

$$
E_{h, 1} \gamma_{0} \xi_{h, 1}^{n}=\xi_{h, 1_{*}}^{n}
$$

Then

$$
\begin{aligned}
a_{1}\left(\xi_{h, 1}^{n}, \xi_{h, 1}^{n}\right)= & a_{1}\left(\xi_{h, 1}^{n}, \xi_{h, 1_{* *}}^{n}\right)-a_{2}\left(\xi_{h, 2}^{n}, E_{h, 2} \gamma_{0} \xi_{h, 1}^{n}\right) \\
& -R_{e} b_{2}\left(u_{h, 2}^{n-1}, u_{h, 2}^{n}, E_{h, 2} \gamma_{0} \xi_{h, 1}^{n}\right)+R_{e} b_{2}\left(u_{h, 2}, u_{h, 2}, E_{h, 2} \gamma_{0} \xi_{h, 1}^{n}\right) \\
& -R_{e} b_{1}\left(u_{h, 1}^{n-1}, u_{h, 1}^{n}, \xi_{h, 1_{*}}^{n}\right)+R_{e} b_{1}\left(u_{h, 1}, u_{h, 1}, \xi_{h, 1_{*}}^{n}\right) .
\end{aligned}
$$

By Lemmas 3.1 and 3.2, we get

$$
\begin{aligned}
\left\|\xi_{h, 1}^{n}\right\|_{1}^{2} & \leq\left\|\xi_{h, 2}^{n}\right\|_{2}\left\|E_{h, 2} \gamma_{0} \xi_{h, 1}^{n}\right\|_{2}+\left\|\xi_{h, 1}^{n}\right\|_{1}\left\|\xi_{h, 1_{* *}}^{n}\right\|_{1}+\varepsilon G_{2}^{n}\left\|E_{h, 2} \gamma_{0} \xi_{h, 1}^{n}\right\|_{2}+\varepsilon G_{1}^{n}\left\|\xi_{h, 1_{*}}^{n}\right\|_{1} \\
& \leq \tau^{\frac{1}{2}}\left\|\xi_{h, 2}^{n}\right\|_{2}\left\|\xi_{h, 1_{*}}^{n}\right\|_{1}+\left\|\xi_{h, 1}^{n}\right\|_{1}\left\|\xi_{h, 1_{* *}}^{n}\right\|_{1}+\tau^{\frac{1}{2}} \varepsilon G_{2}^{n}\left\|\xi_{h, 1_{*}}^{n}\right\|_{1}+\varepsilon G_{1}^{n}\left\|\xi_{h, 1_{*}}^{n}\right\|_{1} \\
& \leq \tau^{\frac{1}{2}}\left\|\xi_{h, 2}^{n}\right\|_{2}\left\|\xi_{h, 1}^{n}\right\|_{1}+\tau^{\frac{1}{2}} \varepsilon G_{2}^{n}\left\|\xi_{h, 1}^{n}\right\|_{1}+2 \varepsilon G_{1}^{n}\left\|\xi_{h, 1}^{n}\right\|_{1} .
\end{aligned}
$$

Then

$$
\left\|\xi_{h, 1}^{n}\right\|_{1} \leq \tau^{\frac{1}{2}}\left\|\xi_{h, 2}^{n}\right\|_{2}+\tau^{\frac{1}{2}} \varepsilon G_{2}^{n}+2 \varepsilon G_{1}^{n}
$$

So

$$
\left\|\xi_{h, 1}^{n}\right\|_{1}^{2} \leq 3 \tau\left\|\xi_{h, 2}^{n}\right\|_{2}^{2}+3 \tau\left(G_{2}^{n}\right)^{2}+12 \varepsilon^{2}\left(G_{1}^{n}\right)^{2}
$$

which implies Lemma 3.5.

Lemma 3.6. Provided $R_{e}$ is sufficiently small, then for every $n \geq 1$,

$$
\left\|\xi_{h, 1}^{n}\right\|_{1} \leq M, \quad\left\|\xi_{h, 2}^{n}\right\|_{2} \leq 2 \sigma^{\frac{1}{2}}\left\|\xi_{h, 1}^{n}\right\|_{1} \leq 2 \sigma^{\frac{1}{2}} M
$$

where $M=\max \left\{\left\|\xi_{h, 1}^{0}\right\|_{1},\left\|\xi_{h, 1}^{1}\right\|_{1}\right\}$. 
Proof. We show this lemma by induction. Assume that $\left\|\xi_{h, 1}^{n}\right\|_{1} \leq M$, we now prove that $\left\|\xi_{h, 1}^{n+1}\right\|_{1} \leq M$. In the first step, we show that if $\left\|\xi_{h, 1}^{n}\right\|_{1} \leq M$, then $\left\|\xi_{h, 2}^{n}\right\|_{2} \leq 2 \sigma^{\frac{1}{2}} M(n \geq 1)$. We will prove this assertion also by induction.

Taking $v=\xi_{h, 2}^{n}$ in (3.9), we have

$$
\begin{aligned}
a_{2}\left(\xi_{h, 2}^{n}, \xi_{h, 2}^{n}\right)= & -a_{1}\left(\xi_{h, 1}^{n}, E_{h, 1} \gamma_{0} \xi_{h, 2}^{n}\right) \\
& -R_{e} b_{2}\left(u_{h, 2}^{n-1}, u_{h, 2}^{n}, \xi_{h, 2}^{n}\right)+R_{e} b_{2}\left(u_{h, 2}, u_{h, 2}, \xi_{h, 2}^{n}\right) \\
& -R_{e} b_{1}\left(u_{h, 1}^{n-1}, u_{h, 1}^{n}, E_{h, 1} \gamma_{0} \xi_{h, 2}^{n}\right)+R_{e} b_{1}\left(u_{h, 1}, u_{h, 1}, E_{h, 1} \gamma_{0} \xi_{h, 2}^{n}\right) .
\end{aligned}
$$

Then

$$
\left\|\xi_{h, 2}^{n}\right\|_{2}^{2} \leq\left\|\xi_{h, 1}^{n}\right\|_{1}\left\|E_{h, 1} \gamma_{0} \xi_{h, 2}^{n}\right\|_{1}+\varepsilon G_{2}^{n}\left\|\xi_{h, 2}^{n}\right\|_{2}+\varepsilon G_{1}^{n}\left\|E_{h, 1} \gamma_{0} \xi_{h, 2}^{n}\right\|_{1} .
$$

On the other hand, using Lemma 3.4,

$$
\left\|E_{h, 1} \gamma_{0} \xi_{h, 2}^{n}\right\|_{1} \leq \sigma^{\frac{1}{2}}\left\|E_{h, 2} \gamma_{0} \xi_{h, 2}^{n}\right\|_{2} \leq \sigma^{\frac{1}{2}}\left\|\xi_{h, 2}^{n}\right\|_{2}+\sigma^{\frac{1}{2}} \varepsilon G_{2}^{n} .
$$

So combining the above two inequalities, we get

$$
\left\|\xi_{h, 2}^{n}\right\|_{2} \leq \sigma^{\frac{1}{2}}\left\|\xi_{h, 1}^{n}\right\|_{1}\left\|\xi_{h, 2}^{n}\right\|_{2}+\varepsilon \sigma^{\frac{1}{2}} G_{2}^{n}\left\|\xi_{h, 1}^{n}\right\|_{1}+\varepsilon G_{2}^{n}\left\|\xi_{h, 2}^{n}\right\|_{2}+\varepsilon \sigma^{\frac{1}{2}} G_{1}^{n}\left\|\xi_{h, 2}^{n}\right\|_{2}+\varepsilon^{2} \sigma^{\frac{1}{2}} G_{1}^{n} G_{2}^{n} .
$$

When $n=1$, then

$$
\left\|\xi_{h, 2}^{1}\right\|_{2}^{2} \leq \sigma^{\frac{1}{2}} M\left\|\xi_{h, 2}^{1}\right\|_{2}+\varepsilon \sigma^{\frac{1}{2}} G_{2}^{1} M+\varepsilon G_{2}^{1}\left\|\xi_{h, 2}^{1}\right\|_{2}+\varepsilon \sigma^{\frac{1}{2}} G_{1}\left\|\xi_{h, 2}^{1}\right\|_{2}+\varepsilon^{2} G_{1}^{1} G_{2}^{1} \sigma^{\frac{1}{2}} .
$$

It is easy to see that

$$
\begin{aligned}
& G_{1}^{1} \leq\left(M+C^{-1} \varepsilon\|f\|_{-1}\right) M+M C^{-1} \varepsilon\|f\|_{-1}, \\
& G_{2}^{1} \leq\left(N+C^{-1} \varepsilon\|f\|_{-1}\right)\left\|\xi_{h, 2}^{1}\right\|_{2}+N C^{-1} \varepsilon\|f\|_{-1},
\end{aligned}
$$

where $N=\left\|\xi_{h, 2}^{0}\right\|_{2}$.

Combining above three inequalities, and by simple manipulation, we can achieve

$$
(1-O(\varepsilon))\left\|\xi_{h, 2}^{1}\right\|_{2}^{2} \leq\left(\sigma^{\frac{1}{2}} M+O(\varepsilon)\right)\left\|\xi_{h, 2}^{1}\right\|_{2}+O(\varepsilon) .
$$

When $\varepsilon \rightarrow 0$, simple calculation can yields

$$
\left\|\xi_{h, 2}^{1}\right\|_{2} \leq 2 \sigma^{\frac{1}{2}} M
$$

Assume the assertion holds for $n-1$, we then have

$$
\begin{aligned}
& G_{1}^{n} \leq\left(M+C^{-1} \varepsilon\|f\|_{-1}\right) M+M C^{-1} \varepsilon\|f\|_{-1}, \\
& G_{2}^{n} \leq\left(2 \sigma^{\frac{1}{2}} M+C^{-1} \varepsilon\|f\|_{-1}\right)\left\|\xi_{h, 2}^{1}\right\|_{2}+2 \sigma^{\frac{1}{2}} M C^{-1} \varepsilon\|f\|_{-1},
\end{aligned}
$$

By (3.13), we have

$$
(1-O(\varepsilon))\left\|\xi_{h, 2}^{n}\right\|_{2}^{2} \leq\left(\sigma^{\frac{1}{2}} M+O(\varepsilon)\right)\left\|\xi_{h, 2}^{n}\right\|_{2}+O(\varepsilon) .
$$


When $\varepsilon \rightarrow 0$, simple calculation can yields

$$
\left\|\xi_{h, 2}^{n}\right\|_{2} \leq 2 \sigma^{\frac{1}{2}} M
$$

We now prove that if $\left\|\xi_{h, 1}^{n}\right\|_{1} \leq M$, then $\left\|\xi_{h, 1}^{n+1}\right\|_{1} \leq M$. From (3.5), we have

$$
\left\|\xi_{h, 1_{*}}^{n+1}\right\|_{1}^{2}=\theta_{n}^{2}\left\|E_{h, 1} \gamma_{0} \xi_{h, 2}^{n}\right\|_{1}^{2}+\left(1-\theta_{n}\right)^{2}\left\|\xi_{h, 1_{*}}^{n}\right\|_{1}^{2}+2 \theta_{n}\left(1-\theta_{n}\right) a_{1}\left(E_{h, 1} \gamma_{0} \xi_{h, 2}^{n}, \xi_{h, 1_{*}}^{n}\right) .
$$

Note that $\theta_{n}\left(1-\theta_{n}\right) \leq \frac{1}{4}$. On the other hand, by Lemma 3.4,

$$
\begin{aligned}
\left\|E_{h, 1} \gamma_{0} \xi_{h, 2}^{n}\right\|_{1}^{2} & \leq \sigma\left\|E_{h, 2} \gamma_{0} \xi_{h, 2}^{n}\right\|_{2}^{2} \\
& \leq \sigma\left\|\xi_{h, 2}^{n}\right\|_{2}^{2}+\varepsilon^{2} \sigma\left(G_{2}^{n}\right)^{2}+2 \varepsilon \sigma G_{2}^{n} .
\end{aligned}
$$

By (3.12), we know that

$$
\begin{aligned}
a_{1}\left(\xi_{h, 1_{*}}^{n}, E_{h, 1} \gamma_{0} \xi_{h, 2}^{n}\right)= & -a_{2}\left(\xi_{h, 2}^{n}, \xi_{h, 2}^{n}\right)-a_{1}\left(\xi_{h, 1_{* *}}^{n}, E_{h, 1} \gamma_{0} \xi_{h, 2}^{n}\right) \\
& -R_{e} b_{2}\left(u_{h, 2}^{n-1}, u_{h, 2}^{n}, \xi_{h, 2}^{n}\right)+R_{e} b_{2}\left(u_{h, 2}, u_{h, 2}, \xi_{h, 2}^{n}\right) \\
& -R_{e} b_{1}\left(u_{h, 2}^{n-1}, u_{h, 1}^{n}, E_{h, 1} \gamma_{0} \xi_{h, 2}^{n}\right)+R_{e} b_{1}\left(u_{h, 1}, u_{h, 1}, E_{h, 1} \gamma_{0} \xi_{h, 2}^{n}\right) .
\end{aligned}
$$

Combining above three results, we can derive that

$$
\begin{aligned}
\left\|\xi_{h, 1_{*}}^{n+1}\right\|_{1}^{2} \leq & \sigma \theta_{n}^{2}\left\|\xi_{h, 2}^{n}\right\|_{2}^{2}+\left(1-\theta_{n}\right)^{2}\left\|\xi_{h, 1_{*}}^{n}\right\|_{1}^{2}-2 \theta_{n}\left(1-\theta_{n}\right)\left\|\xi_{h, 2}^{n}\right\|_{2}^{2} \\
& +\varepsilon^{2} \theta_{n}^{2} \sigma\left(G_{2}^{n}\right)^{2}+2 \varepsilon \sigma \theta_{n}^{2} G_{2}^{n}\left\|\xi_{h, 2}^{n}\right\|_{2} \\
& -2 \theta_{n}\left(1-\theta_{n}\right)\left[a_{1}\left(\xi_{h, 1_{* *}}^{n}, E_{h, 1} \gamma_{0} \xi_{h, 2}^{n}\right)\right. \\
& +R_{e} b_{2}\left(u_{h, 2}^{n-1}, u_{h, 2}^{n}, \xi_{h, 2}^{n}\right)-R_{e} b_{2}\left(u_{h, 2}, u_{h, 2}, \xi_{h, 2}^{n}\right) \\
& \left.+R_{e} b_{1}\left(u_{h, 1}^{n-1}, u_{h, 1}^{n}, E_{h, 1} \gamma_{0} \xi_{h, 2}^{n}\right)-R_{e} b_{1}\left(u_{h, 1}, u_{h, 1}, E_{h, 1} \gamma_{0} \xi_{h, 2}^{n}\right)\right] \\
\leq & \left(4 \sigma^{2} \theta_{n}^{2}+\left(1-\theta_{n}\right)^{2}-\frac{2 \theta_{n}\left(1-\theta_{n}\right)}{3 \tau}\right)\left\|\xi_{h, 1}^{n}\right\|_{1}^{2} \\
& +\frac{1}{2}\left\|\xi_{h, 1_{* *}}^{n}\right\|_{1}\left\|E_{h, 1} \gamma_{0} \xi_{h, 2}^{n}\right\|_{1} \\
& +\varepsilon G_{2}^{n}\left\|\xi_{h, 2}^{n}\right\|_{2}+\varepsilon G_{1}^{n}\left\|E_{h, 1} \gamma_{0} \xi_{h, 2}^{n}\right\|_{1} .
\end{aligned}
$$

Using Lemmas 3.1, 3.2, 3.4 and 3.5 and the induction assumption, by elementary manipulation, we can obtain

$$
\left\|\xi_{h, 1_{*}}^{n+1}\right\|_{1} \leq K\left(\theta_{n}\right)\left\|\xi_{h, 1}^{n}\right\|_{1}+O(\varepsilon)
$$

where $K\left(\theta_{n}\right)=\left(4 \sigma^{2} \theta_{n}^{2}+\left(1-\theta_{n}\right)^{2}-\frac{2 \theta_{n}\left(1-\theta_{n}\right)}{3 \tau}\right)^{\frac{1}{2}}$.

By an elementary calculation, if

$$
\frac{3 \tau+2}{12 \sigma^{2} \tau+3 \tau+2} \hat{=} \theta_{*} \leq \theta_{n}<\theta^{*} \hat{=} \min \left\{\frac{6 \tau+2}{12 \sigma^{2} \tau+3 \tau+2}, 1\right\}
$$

then

$$
0<K\left(\theta_{n}\right) \leq d<1
$$

So by Lemma 3.1, we finally get

$$
\left\|\xi_{h, 1}^{n+1}\right\|_{1} \leq d M+O(\varepsilon)\left\|\xi_{h, 1}^{n+1}\right\|_{1}+O(\varepsilon)
$$


Then

When $\varepsilon \rightarrow 0$, we have

$$
\left\|\xi_{h, 1}^{n+1}\right\|_{1} \leq \frac{d}{1-O(\varepsilon)} M+O(\varepsilon)
$$

We have proved Lemma 3.6.

$$
\left\|\xi_{h, 1}^{n+1}\right\|_{1} \leq M
$$

Theorem 3.1. Assume that $R_{e}$ is sufficiently small and $\theta_{n} \in\left(\theta_{*}, \theta^{*}\right)$, then the D-N velocity sequence $\left\{u_{h, 1}^{n}, u_{h, 2}^{n}\right\}$ converges geometrically to the true solution $u_{h}=\left(u_{h, 1}, u_{h, 2}\right)$.

Proof. From the proof of Lemma 3.6, we know that if $R_{e}$ is sufficiently small, then

$$
\left\|\xi_{h, 2}^{n}\right\|_{2} \leq 2 \sigma^{\frac{1}{2}}\left\|\xi_{h, 1}^{n}\right\|_{1}
$$

Using the above inequality and the same arguments as in the proof of Lemma 3.6, we can prove that

$$
\begin{aligned}
\left\|\xi_{h, 1}^{n+1}\right\|_{1} & \leq d\left\|\xi_{h, 1}^{n}\right\|_{1}+O(\varepsilon)+\varepsilon\left(M+2 C^{-1} \varepsilon\|f\|_{-1}\right)\left\|\xi_{h, 1}^{n+1}\right\|_{1} \\
& \leq(d+O(\varepsilon))\left\|\xi_{h, 1}^{n}\right\|_{1}+O(\varepsilon)\left\|\xi_{h, 1}^{n+1}\right\|_{1} .
\end{aligned}
$$

Then

$$
\left\|\xi_{h, 1}^{n+1}\right\|_{1} \leq \frac{d+O(\varepsilon)}{1-O(\varepsilon)}\left\|\xi_{h, 1}^{n}\right\|_{1} .
$$

Based on the above inequality, we can easily see that if $R_{e}$ is sufficiently small, then $\xi_{h, 1}^{n}$ converges geometrically to zero. On the other hand, because of (3.13), we know that $\xi_{h, 2}^{n}$ also converges geometrically to zero.

Theorem 3.2. Assume that $R_{e}$ is sufficiently small and $\theta_{n} \in\left(\theta_{*}, \theta^{*}\right)$, then the D-N pressure sequence $\left\{p_{h, 1}^{n}, p_{h, 2}^{n}\right\}$ converges to the true solution $\left(p_{h, 1}, p_{h, 2}\right)$.

Proof. Let $\beta_{k}(k=1,2)$ be the constant in the inf-sup condition (2.5) restricted to $\Omega_{k}$. Using (3.3),

$$
\begin{aligned}
\left\|\eta_{h, 1}^{n}\right\|_{0} & \leq \beta_{1} \sup _{v \in V_{h, 1}} \frac{c_{1}\left(v, \eta_{h, 1}^{n}\right)}{\|v\|_{1}} \\
& \leq \beta_{1} \sup _{v \in V_{h, 1}} \frac{\left|-\frac{1}{R_{e}} a_{1}\left(\xi_{h, 1}^{n}, v\right)-b_{1}\left(u_{h, 1}^{n-1}, u_{h, 1}^{n}, v\right)+b_{1}\left(u_{h, 1}, u_{h, 1}, v\right)\right|}{\|v\|_{1}} \\
& \leq \beta_{1}\left[R_{e}^{-1}\left\|\xi_{h, 1}^{n}\right\|_{1}+\left(\left\|\xi_{h, 1}^{n-1}\right\|_{1}+\left\|u_{h}\right\|\right)\left\|\xi_{h, 1}^{n}\right\|_{1}+\left\|\xi_{h, 1}^{n-1}\right\|_{1}\left\|u_{h}\right\|\right] \\
& \leq \beta_{1}\left[\left(R_{e}^{-1} M+C^{-1} \varepsilon\|f\|_{-1}\right)\left\|\xi_{h, 1}^{n}\right\|_{1}+C^{-1} \varepsilon\|f\|_{-1}\left\|\xi_{h, 1}^{n-1}\right\|_{1}\right]
\end{aligned}
$$

which means that $\eta_{h, 1}^{n}$ converges to zero.

Similarly, based on (3.9), we have

$$
\begin{aligned}
\left\|\eta_{h, 2}^{n}\right\|_{0} \leq & \beta_{2} \sup _{v \in V_{h, 2}} \frac{c_{2}\left(v, \eta_{h, 2}^{n}\right)}{\|v\|_{2}} \\
\leq & \beta_{2} \sup _{v \in V_{h, 2}}\left[R_{e}^{-1} a_{2}\left(\xi_{h, 2}^{n}, v\right)-b_{2}\left(u_{h, 2}^{n-1}, u_{h, 2}^{n}, v\right)+b_{2}\left(u_{h, 2}, u_{h, 2}, v\right)\right. \\
& -R_{e}^{-1} a_{1}\left(\xi_{h, 1}^{n}, E_{h, 1} \gamma_{0} v\right)-b_{1}\left(u_{h, 1}^{n-1}, u_{h, 1}^{n}, E_{h, 1} \gamma_{0} v\right) \\
& \left.+b_{1}\left(u_{h, 1}, u_{h, 1}, E_{h, 1} \gamma_{0} v\right)\right] /\|v\|_{2} \\
\leq & \beta_{2} \sup _{v \in V_{h, 2}}\left[R_{e}^{-1}\left\|\xi_{h, 2}^{n}\right\|_{2}\|v\|_{2}+C G_{2}^{n}\|v\|_{2}\right. \\
& \left.+R_{e}^{-1}\left\|\xi_{h, 1}^{n}\right\|_{1}\left\|E_{h, 1} \gamma_{0} v\right\|_{1}+C G_{1}^{n}\left\|E_{h, 1} \gamma_{0} v\right\|_{1}\right] /\|v\|_{2} .
\end{aligned}
$$


Using a result in [16] and a trace inequality, we have

$$
\left\|E_{h, 1} \gamma_{0} v\right\|_{1} \leq C_{3}\left\|\gamma_{0} v\right\|_{H_{00}^{\frac{1}{2}}(\Gamma)} \leq C_{3} C_{4}\|v\|_{2} .
$$

Combining the above two inequalities, we obtain

$$
\begin{aligned}
\left\|\eta_{h, 2}^{n}\right\|_{0} \leq & \beta_{2}\left[\left(R e^{-1}+2 \sigma^{\frac{1}{2}} M+C^{-1} \varepsilon\|f\|_{-1}\right)\left\|\xi_{h, 2}^{n}\right\|_{2}+2 \sigma^{\frac{1}{2}} M C^{-1} \varepsilon\|f\|_{-1}\left\|\xi_{h, 2}^{n-1}\right\|_{2}\right. \\
& \left.+\left(R_{e}^{-1} M+C^{-1} \varepsilon\|f\|_{-1}\right)\left\|\xi_{h, 1}^{n}\right\|_{1}+C^{-1} \varepsilon\|f\|_{-1}\left\|\xi_{h, 1}^{n-1}\right\|_{1}\right] .
\end{aligned}
$$

Again, this implies that $\eta_{h, 2}^{n}$ converges geometrically to zero.

\section{Other Dirichlet-Neumann iterative Algorithms}

In this section, we give other version of the D-N iterative algorithm where only linear Stokes need to be solved in each subdomain. Such kind of algorithm is obviously easier to implement.

Algorithm 4.1. For any $g_{h}^{0} \in \hat{\Phi}_{h}$ and $u_{h, k}^{0} \in V_{h, k}, k=1,2$, define the two sequences $\left(u_{h, 1}^{n}, p_{h, 1}^{n}\right) \in V_{h, 1} \times M_{h, 1}$ and $\left(u_{h, 2}^{n}, p_{h, 2}^{n}\right) \in V_{h, 2} \times M_{h, 2}$ as follows:

$$
\left\{\begin{aligned}
\frac{1}{R_{e}} a_{1}\left(u_{h, 1}^{n}, v\right)+c_{1}\left(v, p_{h, 1}^{n}\right) & =(f, v)_{1}-b_{1}\left(u_{h, 1}^{n-1}, u_{h, 1}^{n-1}, v\right), \quad \forall v \in V_{h, 1}^{0} \\
c_{1}\left(u_{h, 1}^{n}, q\right) & =0, \quad \forall q \in M_{h, 1} \cap L_{0}^{2}(\Omega) \\
\gamma_{0} u_{h, 1}^{n} & =g_{h}^{n-1}, \quad \text { on } \Gamma \\
\int_{\Omega_{1}} p_{h, 1} \mathrm{~d} x & =\int_{\Omega_{2}} p_{h, 2} \mathrm{~d} x
\end{aligned}\right.
$$

and

$$
\left\{\begin{aligned}
\frac{1}{R_{e}} a_{2}\left(u_{h, 2}^{n}, v\right)+c_{2}\left(v, p_{h, 2}^{n}\right)= & (f, v)_{2}+\left(f, \rho_{h, 1} \gamma_{0} v\right)_{1}-b_{2}\left(u_{h, 2}^{n-1}, u_{h, 2}^{n-1}, v\right) \\
& -\frac{1}{R_{e}} a_{1}\left(u_{h, 1}^{n}, \rho_{h, 1} \gamma_{0} v\right)-b_{1}\left(u_{h, 1}^{n-1}, u_{h, 1}^{n-1}, \rho_{h, 1} \gamma_{0} v\right) \\
& -c_{1}\left(\rho_{h, 1} \gamma_{0} v, p_{h, 1}^{n}\right), \quad \forall v \in V_{h, 2} \\
c_{2}\left(u_{h, 2}^{n}, q\right)= & 0, \quad \forall q \in M_{h, 2},
\end{aligned}\right.
$$

where we have set

$$
g_{h}^{n}=\theta_{n} \gamma_{0} u_{h, 2}^{n}+\left(1-\theta_{n}\right) g_{h}^{n-1} .
$$

Similar to the previous section, we can prove

Lemma 4.1. Provided $R_{e}$ is sufficiently small, then for every $n \geq 1$,

$$
\left\|\xi_{h, 1}^{n}\right\|_{1} \leq M, \quad\left\|\xi_{h, 2}^{n}\right\|_{2} \leq 3 \sigma^{\frac{1}{2}} M
$$

where $M=\left\|\xi_{h, 1}^{1}\right\|_{1}$.

Theorem 4.1. Assume that $R_{e}$ and $\theta_{n}$ are sufficiently small, then the linear $D-N$ velocity sequence in Algorithm $4.1\left\{u_{h, 1}^{n}, u_{h, 2}^{n}\right\}$ converges to the true solution $u_{h}=\left(u_{h, 1}, u_{h, 2}\right)$.

Proof. The proof is similar to before, we only give a skeleton of the proof.

Using a similar argument as in the proof of Lemma 3.6, we have

$$
\left\|\xi_{h, 1_{*}}^{n+1}\right\|_{1}^{2} \leq \sigma \theta_{n}^{2}\left\|\xi_{h, 2}^{n}\right\|_{2}^{2}+\left(1-\theta_{n}\right)^{2}\left\|\xi_{h, 1_{*}}^{n}\right\|_{1}^{2}-\frac{2 \theta\left(1-\theta_{n}\right)}{3 \tau}\left\|\xi_{h, 1}^{n}\right\|_{1}^{2}+O(\varepsilon) .
$$


So

$$
\begin{aligned}
\left\|\xi_{h, 1}^{n+1}\right\|_{1}^{2} & =\left\|\xi_{h, 1_{1}}^{n+1}\right\|_{1}^{2}+\left\|\xi_{h, 1_{* *}}^{n+1}\right\|_{1}^{2} \\
& \leq \sigma \theta_{n}^{2}\left\|\xi_{h, 2}^{n}\right\|_{1}^{2}+\delta\left\|\xi_{h, 1}^{n}\right\|_{1}^{2}+O(\varepsilon)
\end{aligned}
$$

where $\delta \hat{=}\left(1-\theta_{n}\right)^{2}-\frac{2 \theta\left(1-\theta_{n}\right)}{3 \tau}$.

On the other hand,

$$
\begin{aligned}
\left\|\xi_{h, 2}^{n+1}\right\|_{2}^{2} & \leq\left\|\xi_{h, 1}^{n+1}\right\|_{1}\left\|E_{h, 1} \gamma_{0} \xi_{h, 2}^{n+1}\right\|_{1}+O(\varepsilon) \\
& \leq \sigma^{\frac{1}{2}}\left\|\xi_{h, 1}^{n+1}\right\|_{1}\left\|\xi_{h, 2}^{n+1}\right\|_{2}+O(\varepsilon) \\
& \leq \frac{\sigma}{2}\left\|\xi_{h, 1}^{n+1}\right\|_{1}^{2}+\frac{1}{2}\left\|\xi_{h, 2}^{n+1}\right\|_{2}^{2}+O(\varepsilon)
\end{aligned}
$$

Then,

$$
\begin{aligned}
\left\|\xi_{h, 2}^{n+1}\right\|_{2}^{2} & \leq \sigma\left\|\xi_{h, 1}^{n+1}\right\|_{1}^{2}+O(\varepsilon) \\
& \leq \sigma^{2} \theta_{n}^{2}\left\|\xi_{h, 2}^{n}\right\|_{1}^{2}+\sigma \delta\left\|\xi_{h, 1}^{n}\right\|_{1}^{2}+O(\varepsilon) .
\end{aligned}
$$

Finally,

$$
\left[\begin{array}{l}
\left\|\xi_{h, 1}^{n+1}\right\|_{1}^{2} \\
\left\|\xi_{h, 2}^{n+1}\right\|_{2}^{2}
\end{array}\right] \leq\left[\begin{array}{cc}
\delta & \theta_{n}^{2} \sigma \\
\sigma \delta & \sigma^{2} \theta_{n}^{2}
\end{array}\right]\left[\begin{array}{c}
\left\|\xi_{h, 1}^{n}\right\|_{1}^{2} \\
\left\|\xi_{h, 2}^{n}\right\|_{2}^{2}
\end{array}\right]+O(\varepsilon)\left[\begin{array}{l}
1 \\
1
\end{array}\right]
$$

Now $\rho(A)=\delta+\sigma^{2} \theta_{n}^{2}+o(1)$, as $\epsilon \rightarrow 0$ where

$$
A=\left[\begin{array}{cc}
\delta & \theta_{n}^{2} \sigma \\
\sigma \delta & \sigma^{2} \theta_{n}^{2}
\end{array}\right]
$$

and $\rho(A)$ is the spectral radius of $A$. By selecting the approximate $(\epsilon=0)$ optimal value $\theta_{n}=\frac{3 \tau+1}{3 \tau \sigma^{2}+3 \tau+2}, \rho(A)<1$. So we know that the linear D-N velocity sequence $\left\{u_{h, 1}^{n}, u_{h, 2}^{n}\right\}$ converges to the true solution $\left(u_{h, 1}, u_{h, 2}\right)$.

Based on Theorem 4.1 and a similar argument as before, we have

Theorem 4.2. Assume that $R_{e}$ and $\theta_{n}$ are sufficiently small, then the linear $D-N$ pressure sequence in Algorithm $4.1\left\{p_{h, 1}^{n}, p_{h, 2}^{n}\right\}$ converges to the true solution $\left(p_{h, 1}, p_{h, 2}\right)$.

\section{The Neumann-Neumann iterative Algorithm}

Let us consider now another well-known nonoverlapping DDM, the Neumann-Neumann (N-N) iterative algorithm, for solving (2.6). We make an additional assumption that $\Omega_{1}$ is also not an interior subdomain.

Similar to the above section, let $V_{h} \subset V$ and $M_{h} \subset M$ be finite element spaces. We also assume that the pressure space $M_{h}$ consists of functions which are discontinuous across inter-element boundaries. Moreover, we assume that the pressure has a zero mean value. Similar to Section 3, we can also define the corresponding subspaces $V_{h, k}, M_{h, k}, k=1,2$. However, we don't assume that functions in $M_{h, 1}$ vanish at a point in $\Omega_{1}$.

Following [17], we may define the N-N iterative algorithm as follows: For any $\lambda_{h}^{0} \in \hat{\Phi}_{h}$ and $p_{h, k}^{0} \in M_{h, k} \cap$ $L_{0}^{2}\left(\Omega_{k}\right), k=1,2$, the sequences $\left(u_{h, k}^{n}, p_{h, k}^{n}\right) \in V_{h, k} \times M_{h, k} \cap L_{0}^{2}\left(\Omega_{k}\right)$ are defined by

$$
\left\{\begin{aligned}
\frac{1}{R_{e}} a_{1}\left(u_{h, 1}^{n}, v\right)+b_{1}\left(u_{h, 1}^{n}, u_{h, 1}^{n}, v\right)+c_{1}\left(v, p_{h, 1}^{n}\right) & =(f, v)_{1}, \quad \forall v \in V_{h, 1}^{0} \\
c_{1}\left(u_{h, 1}^{n}, q\right) & =0, \quad \forall q \in M_{h, 1} \cap L_{0}^{2}\left(\Omega_{1}\right) \\
\gamma_{0} u_{h, 1}^{n} & =\lambda_{h}^{n-1}, \quad \text { on } \Gamma, \\
\int_{\Omega_{1}} p_{h, 1}^{n} \mathrm{~d} x & =-\int_{\Omega_{2}} p_{h, 2}^{n-1} \mathrm{~d} x,
\end{aligned}\right.
$$


and

$$
\left\{\begin{aligned}
\frac{1}{R_{e}} a_{2}\left(u_{h, 2}^{n}, v\right)+b_{2}\left(u_{h, 2}^{n}, u_{h, 2}^{n}, v\right)+c_{2}\left(v, p_{h, 2}^{n}\right) & =(f, v)_{2}, \quad \forall v \in V_{h, 2}^{0} \\
c_{2}\left(u_{h, 2}^{n}, q\right) & =0, \quad \forall q \in M_{h, 2} \cap L_{0}^{2}\left(\Omega_{2}\right) \\
\gamma_{0} u_{h, 2}^{n} & =\lambda_{h}^{n-1}, \text { on } \Gamma, \\
\int_{\Omega_{2}} p_{h, 2}^{n} \mathrm{~d} x & =-\int_{\Omega_{1}} p_{h, 1}^{n-1} \mathrm{~d} x,
\end{aligned}\right.
$$

and $\left(\psi_{h, k}^{n}, \pi_{h, k}^{n}\right) \in V_{h, k} \times M_{h, k}$, for $k=1,2$, are defined by

$$
\left\{\begin{aligned}
& \frac{1}{R_{e}} a_{k}\left(\psi_{h, k}^{n}, v\right)+b_{k}\left(\psi_{h, k}^{n}, \psi_{h, k}^{n}, v\right)+c_{k}\left(v, \pi_{h, k}^{n}\right)=\quad \sum_{j=1}^{2}\left(f, \rho_{h, j} \gamma_{0} v\right)_{j} \\
&-a_{j}\left(u_{h, j}^{n}, \rho_{h, j} \gamma_{0} v\right) \\
&-b_{j}\left(u_{h, j}^{n}, u_{h, j}^{n}, \rho_{h, j} \gamma_{0} v\right) \\
&-c_{j}\left(\rho_{h, j} \gamma_{0} v, u_{h, j}^{n}\right), \quad \forall v \in V_{h, k} \\
& c_{k}\left(\psi_{h, k}^{n}, q\right)=0, \quad \forall q \in M_{h, k} .
\end{aligned}\right.
$$

Set

$$
\lambda_{h}^{n}=\lambda_{h}^{n-1}+\theta\left(\gamma_{0} \psi_{h, 1}^{n}+\gamma_{0} \psi_{h, 2}^{n}\right) .
$$

Using a similar argument as before, we can prove

Theorem 5.1. Assume that $R_{e}$ is sufficiently small and $\theta$ is suitably chosen, then the $N$ - $N$ velocity iterates $\left\{u_{h, 1}^{n}, u_{h, 2}^{n}\right\}$ converge geometrically to the true solution $\left(u_{h, 1}, u_{h, 2}\right)$.

and

Theorem 5.2. Assume that $R_{e}$ is sufficiently small and $\theta$ is suitably chosen, then the $N$ - $N$ pressure iterates $\left\{p_{h, 1}^{n}, p_{h, 2}^{n}\right\}$ converge geometrically to the true solution $\left(p_{h, 1}, p_{h, 2}\right)$.

\section{Numerical RESULtS}

For a small Reynolds' number, the steady Navier-Stokes equations can be considered as a Stokes problem perturbed by the nonlinear convection term. In this section, we shall illustrate the behavior of the linear D-N Algorithm 4.1 developed in Section 4. In this situation, the computer implementation is simply one for the Stokes problem on each subdomains, for which several finite element methods are available.

Our choice of method is the Taylor-Hood $P_{2}-P_{1}$ triangular elements. More precisely, we discretize the velocities by piecewise quadratic basis functions, and the pressure by piecewise linear basis functions. The nodes for the velocities are the vertices and the midpoints of edges of the triangles; those for the pressure are the vertices of triangles.

On each subdomains, the finite element discretization of the Stokes problem in Algorithm 4.1 leads to the saddle point problem

$$
\left(\begin{array}{cc}
A_{h} & B_{h}^{T} \\
B_{h} & 0
\end{array}\right)\left(\begin{array}{c}
u_{h} \\
p_{h}
\end{array}\right)=\left(\begin{array}{c}
G_{h} \\
0
\end{array}\right)
$$

where $A_{h}=\left(a\left(\phi_{j}, \phi_{k}\right)\right), B_{h}=\left(c\left(\phi_{j}, \psi_{k}\right)\right)$, and $G_{h}=\left(\left(g, \phi_{j}\right)\right), \phi_{j}$ and $\psi_{k}$ being the piecewise quadratic and piecewise linear nodal bases, respectively. The solution to the above saddle point problem can be obtained by a straightforward elimination procedure:

$$
\begin{aligned}
& p_{h}=\left(B_{h} A_{h}^{-1} B_{h}^{T}\right)^{-1} B_{h} A_{h}^{-1} G_{h}, \\
& u_{h}=A_{h}^{-1}\left(G_{h}-B_{h}^{T} p_{h}\right) .
\end{aligned}
$$

Our assumption that the inf-sup condition (2.5) be satisfied ensures the invertibility of $B_{h} A_{h}^{-1} B_{h}^{T}$. Thus, the above elimination procedure makes sense. 


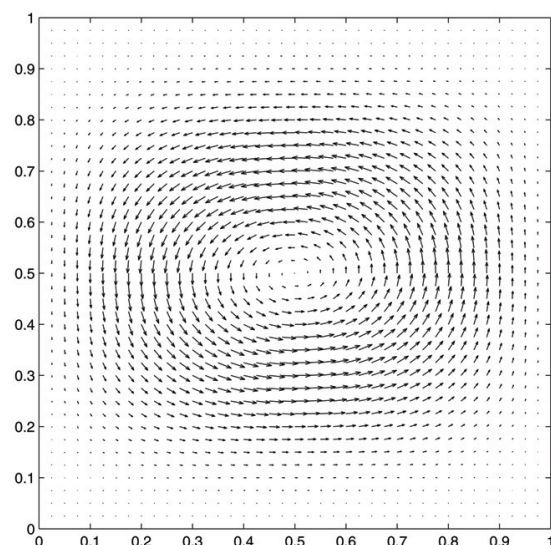

FiguRE 6.1. Flow field $\left(u_{h}, v_{h}\right)$.



Figure 6.2. Pressure contours.

Now for the two-subdomain problem with $\Omega=\Omega_{1} \cup \Omega_{2}$, denote by $A_{h, i}, B_{h, i}$, and $G_{h, i}^{n}$ the corresponding quantities for the subdomain $i, i=1,2$. The linear Dirichlet-Neumann iterative algorithm then becomes the iteration of the following sequence of steps:

$$
\left(\begin{array}{cc}
A_{h, 1} & B_{h, 1}^{T} \\
B_{h, 1} & 0
\end{array}\right)\left(\begin{array}{c}
u_{h, 1}^{n} \\
p_{h, 1}^{n}
\end{array}\right)=\left(\begin{array}{c}
G_{h, 1}^{n} \\
0
\end{array}\right)
$$

where $\left.u_{h, 1}^{n}\right|_{\partial \Omega_{1} \backslash \Gamma}=0,\left.u_{h, 1}^{n}\right|_{\Gamma}=g_{h}^{n-1}$,

$$
\left(\begin{array}{cc}
A_{h, 2} & B_{h, 2}^{T} \\
B_{h, 2} & 0
\end{array}\right)\left(\begin{array}{c}
u_{h, 2}^{n} \\
p_{h, 2}^{n}
\end{array}\right)=\left(\begin{array}{c}
G_{h, 2}^{n} \\
0
\end{array}\right)
$$

where $\left.u_{h, 2}^{n}\right|_{\partial \Omega_{2} \backslash \Gamma}=0$, and

$$
g_{h}^{n}=\left.\theta_{n} u_{h, 2}^{n}\right|_{\Gamma}+\left(1-\theta_{n}\right) g_{h}^{n-1},
$$

where $G_{h, 1}^{n}, G_{h, 2}^{n}$ are associated with the right hand side terms of the first and fifth equalities in the Algorithm 4.1 .

To get the iterations started, we set $u_{h, 1}^{0}=u_{h, 2}^{0}=0$ and $g_{h}^{0}=0$. The Dirichlet boundary conditions for $u_{h, 1}^{n}$ and $u_{h, 2}^{n}$ are easily enforced by explicit elimination, which results in a reduced saddle point problem with a modified right hand side to which the elimination procedure discussed above applies.

Since only $\nabla p$ appears in the Navier-Stokes equations, the pressure $p$ is determined up to an additive constant. For pressure continuous across the interface $\Gamma$, we choose a point $P$ on $\Gamma$ and enforce that $p(P)=0$.

It is convenient to express the flow field in terms of a stream function $\psi=\psi(x, y)$, which determines the velocities $(u, v)=\left(\psi_{y},-\psi_{x}\right)$. In the test cases presented below, a uniform grid with 20 triangles in each spatial direction was used. The unit square $\Omega=(0,1)^{2}$ was divided into two non-overlapping subdomains $\Omega_{1}=\left(0, \frac{1}{2}\right) \times(0,1)$ and $\Omega_{2}=\left(\frac{1}{2}, 1\right) \times(0,1)$, with the interface $\Gamma=\left\{\frac{1}{2}\right\} \times[0,1]$. The point $P$ at which $p=0$ was chosen to be $\left(\frac{1}{2}, \frac{1}{2}\right)$. We took $\theta_{n}=0.2$.

Test case 1: The forcing term $f$ was chosen so that $\psi=x^{2}(1-x)^{2} y^{4}(1-y)^{4}$ and $p=x^{4}(1-x)^{4} y^{2}(1-y)^{2}-2^{-12}$ at $R_{e}=100$. See Figures 6.1 and 6.2 .

Test case 2: $\psi=x^{4} y^{4}(1-x)^{2}(1-y)^{2}$ and $p=\left(x-\frac{1}{2}\right)^{2}-\left(y-\frac{1}{2}\right)^{2}, R_{e}=100$. See Figures 6.3 and 6.4 .

The $H^{1}$ and $L^{2}$ norms of the error $u_{h}^{n}-u_{h}$ are given by

$$
\left\|u_{h}^{n}-u_{h}\right\|_{H^{1}}=\sqrt{\left\|u_{h, 1}^{n}-u_{h, 1}\right\|_{H^{1}}^{2}+\left\|u_{h, 2}^{n}-u_{h, 2}\right\|_{H^{1}}^{2}}
$$




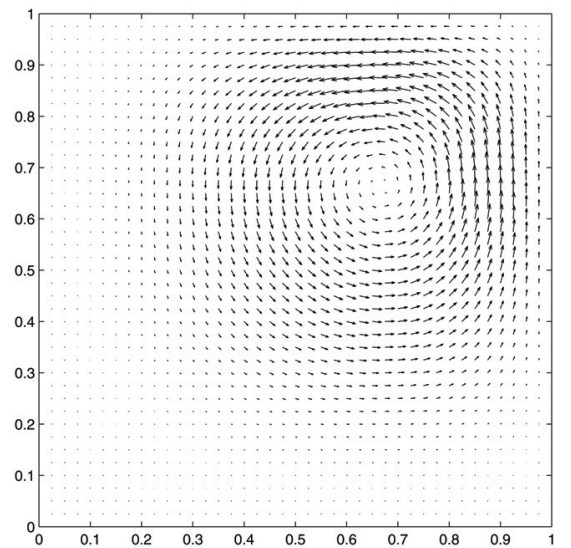

FiguRE 6.3. Flow field $\left(u_{h}, v_{h}\right)$.

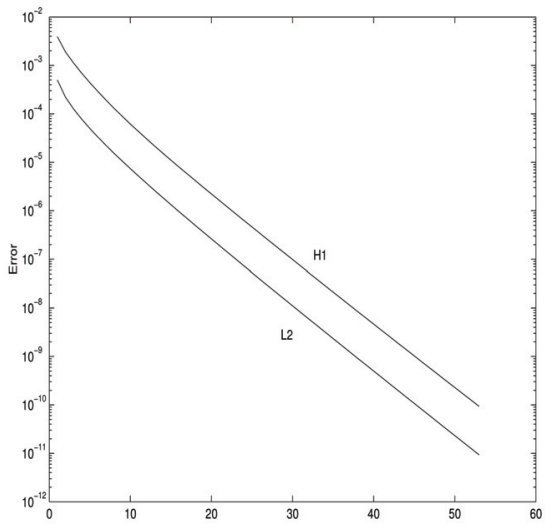

Figure 6.5. Plots of errors for test case 1.

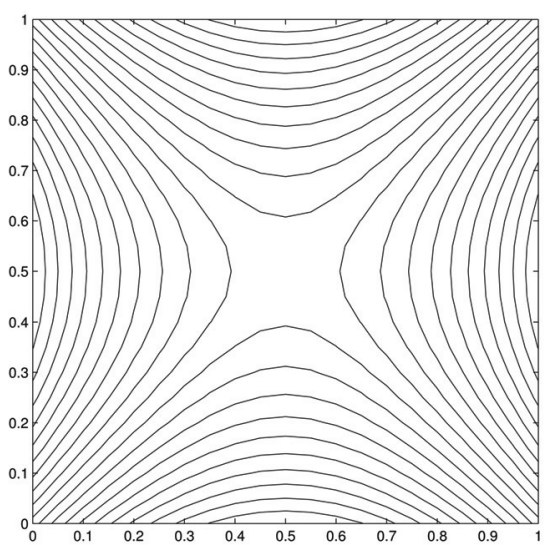

FiguRE 6.4. Pressure contours.

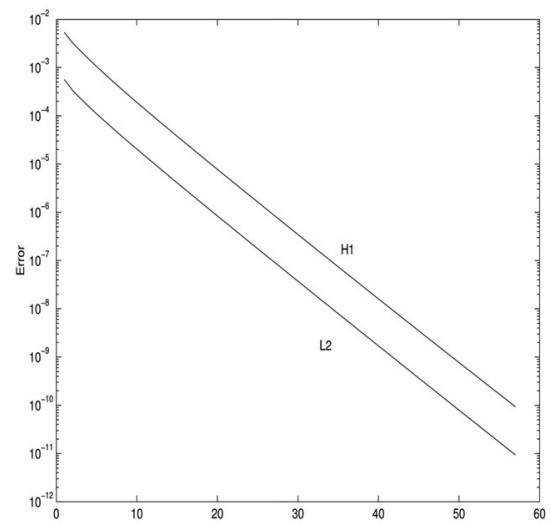

Figure 6.6. Plots of errors for test case 2 .

and

$$
\left\|u_{h}^{n}-u_{h}\right\|_{L^{2}}=\sqrt{\left\|u_{h, 1}^{n}-u_{h, 1}\right\|_{L^{2}}^{2}+\left\|u_{h, 2}^{n}-u_{h, 2}\right\|_{L^{2}}^{2}} .
$$

Plots of these errors versus $n$ for test cases 1 and 2 are given in Figures 6.5 and 6.6, respectively. It is clear that the convergence $\left(u_{h, 1}^{n}, u_{h, 2}^{n}\right) \rightarrow u_{h}=\left(u_{h, 1}, u_{h, 2}\right)$, as established in Theorem 4.1, is honored.

Acknowledgements. We thank the anonymous referees for many constructive comments and suggestions which led to an improved presentation of this paper.

\section{REFERENCES}

[1] J. Cahouet, On some difficulties occurring in the simulation of incompressible fluid flows by domain decomposition methods, in Proc. of the First International Symposium On Domain Decomposition Methods for Partial Differential Equations, R. Glowinski, G. Golub, G. Meurant and J. Periaux Eds., SIAM, Philadelphia, PA (1988).

[2] X.C Cai, D.E. Keyes and V. Venkatakrishnan, Newton-Krylov-Schwarz: An implicit solver for CFD, in Proc. of the Eighth International Conference on Domain Decomposition Methods in Science and Engineering, R. Glowinski, J. Periaux, Z.C. Shi and O.B. Widlund Eds., Wiley, Strasbourg (1997).

[3] T.F. Chan and T.P. Mathew, Domain decomposition algorithm. Acta Numerica (1994) 61-143.

[4] P.G. Ciarlet, The Finite Element Method for Elliptic Problems. North-Holland, Amsterdam (1978). 
[5] Q.V. Dinh, R. Glowinski, J. Periaux and G. Terrasson, On the coupling of viscous and inviscid models for incompressible fluid flows via domain decomposition, in Proc. the First International Symposium On Domain Decomposition Methods for Partial Differential Equations, R. Glowinski, G. Golub, G. Meurant and J. Periaux Eds., SIAM, Philadelphia, PA (1988).

[6] L. Fatone, P. Gervasio and A. Quarteroni, Multimodels for incompressible flows. J. Math. Fluid Dynamics 2 (2000) 126-150.

[7] M. Fortin and R. Aboulaich, Schwarz's Decomposition Method for Incompressible Flow Problems, in Proc. of the First International Symposium On Domain Decomposition Methods for Partial Differential Equations, R. Glowinski, G. Golub, G. Meurant and J. Periaux Eds., SIAM, Philadelphia, PA (1988).

[8] V. Girault and P.A. Raviart, Finite Element Methods for Navier-Stokes Equations, Theory and Algorithms. Spring-Verlag, Berlin (1986).

[9] M. Gunzburger and H.K. Lee, An optimization-based domain decomposition method for the Navier-Stokes equations. SIAM J. Numer. Anal. 37 (2000) 1455-1480.

[10] M. Gunzburger and R. Nicolaides, On substructuring algorithms and solution techniques for numerical approximation of partial differential equations. Appl. Numer. Math. 2 (1986) 243-256.

[11] P. Le Tallec, Domain decomposition methods in computational mechanics. Comput. Mech. Adv. 1 (1994) 121-220.

[12] P.L. Lions, On the Schwarz alternating method, in Proc. of the First International Symposium on Domain Decomposition Methods for Partial Differential Equations, R. Glowinski, G.H. Golub, G.A. Meurant and J. Periaux Eds., SIAM, Philadelphia (1988) $1-42$.

[13] S.H. Lui, On Schwarz alternating methods for nonlinear PDEs. SIAM J. Sci. Comput. 21 (2000) $1506-1523$.

[14] S.H. Lui, On Schwarz alternating methods for the incompressible Navier-Stokes equations. SIAM J. Sci. Comput. 22 (2001) 1974-1986.

[15] S.H. Lui, On linear monotone iteration and Schwarz methods for nonlinear elliptic PDEs. Numer. Math. 93 (2002) $109-129$.

[16] L.D. Marini and A. Quarteroni, A relaxation procedure for domain decomposition methods using finite elements. Numer. Math. 55 (1989) 575-598.

[17] A. Quarteroni and A. Valli, Domain Decomposition Methods for Partial Differential Equations. Oxford Science Publications (1999).

[18] B.F. Smith, P.E. Bjorstad and W.D. Gropp, Domain Decomposition: Parallel Multilevel Algorithms for Elliptic Partial Differential Equations. Cambridge University Press, Cambridge, UK (1996).

[19] R. Teman, The Navier-Stokes Equations, Theory and Numerical Analysis. North-Holland, Amsterdam (1977).

[20] J. Xu and J. Zou, Some nonoverlapping domain decomposition methods. SIAM Rev. 40 (1998) $867-914$.

To access this journal online:

www.edpsciences.org 